\title{
SCIENTIFIC REPORTS

\section{OPEN Early Life Supraphysiological Levels of Oxygen Exposure Permanently Impairs Hippocampal Mitochondrial Function}

Received: 8 April 2019

Published online: 16 September 2019

\begin{abstract}
Manimaran Ramani ${ }^{1}$, Kiara Miller ${ }^{1}$, Jamelle Brown ${ }^{1}$, Ranjit Kumar ${ }^{2}$, Jegen Kadasamy ${ }^{1}$, Lori McMahon ${ }^{3,4}$, Scott Ballinger ${ }^{5}$ \& Namasivayam Ambalavanan $\mathbb{D}^{1,3}$

Preterm infants requiring prolonged oxygen therapy often develop cognitive dysfunction in later life. Previously, we reported that 14-week-old young adult mice exposed to hyperoxia as newborns had spatial and learning deficits and hippocampal shrinkage. We hypothesized that the underlying mechanism was the induction of hippocampal mitochondrial dysfunction by neonatal hyperoxia. C57BL/6J mouse pups were exposed to 85\% oxygen or room air from P2-P14. Hippocampal proteomic analysis was performed in young adult mice (14 weeks). Mitochondrial bioenergetics were measured in neonatal (P14) and young adult mice. We found that hyperoxia exposure reduced mitochondrial ATPlinked oxygen consumption and increased state 4 respiration linked proton leak in both neonatal and young adult mice while complex I function was decreased at P14 but increased in young adult mice. Proteomic analysis revealed that hyperoxia exposure decreased complex I NDUFB8 and NDUFB11 and complex IV 7B subunits, but increased complex III subunit 9 in young adult mice. In conclusion, neonatal hyperoxia permanently impairs hippocampal mitochondrial function and alters complex I function. These hippocampal mitochondrial changes may account for cognitive deficits seen in children and adolescents born preterm and may potentially be a contributing mechanism in other oxidative stress associated brain disorders.
\end{abstract}

Many extremely preterm infants often require prolonged periods of supraphysiological oxygen (hyperoxia) exposure for their survival. In addition, even preterm infants not receiving supplemental oxygen are exposed to a relatively hyperoxemic environment compared to the hypoxemic normal intrauterine environment $\left(\mathrm{PO}_{2} 25-35 \mathrm{~mm}\right.$ $\mathrm{Hg}$ ) during a critical developmental period for many organ systems. Preterm infants who require prolonged periods of oxygen supplementation are at higher risk of morbidities such as retinopathy of prematurity ${ }^{1,2}$ and chronic lung disease like bronchopulmonary dysplasia (BPD) ${ }^{3,4}$, probably as a consequence of chronic oxidative stress (OS). Children with BPD frequently exhibit deficits in executive function and cognition even in the absence of apparent brain injuries such as intraventricular hemorrhage and periventricular leukomalacia ${ }^{5-8}$. Although direct effects of OS and lung injury-induced systemic inflammation on the developing brain have been considered as possible etiologies, the exact mechanism(s) by which children with BPD develop cognitive dysfunction despite no apparent brain injury is not known.

Although the long-term detrimental effects of early hyperoxia exposure on lung development and function have been studied in depth, little is known about long-term effect of early hyperoxia exposure on brain development and function. Previously, we have shown that in $\mathrm{C} 57 \mathrm{BL} / 6 \mathrm{~J}$ mice, hyperoxia $\left(85 \%\right.$ oxygen $\left.\left[\mathrm{O}_{2}\right]\right)$ exposure during the neonatal period (P2-14) (neonatal hyperoxia) leads to spatial memory and learning deficits, increased exploratory behavior, and shrinkage of area CA1 of the hippocampus when assessed at young adult age (14 weeks) ${ }^{9}$. Recently, our proteomic analysis of hippocampal homogenates from neonatal mice (P14) exposed

${ }^{1}$ Departments of Pediatrics, University of Alabama at Birmingham, Birmingham, AL, 35233, USA. ${ }^{2}$ Departments of Bioinformatics, University of Alabama at Birmingham, Birmingham, AL, 35233, USA. ${ }^{3}$ Departments of cell, Developmental, and Integrative Biology, University of Alabama at Birmingham, Birmingham, AL, 35233, USA. ${ }^{4}$ Departments of Neurobiology, University of Alabama at Birmingham, Birmingham, AL, 35233, USA. ${ }^{5}$ Departments of Pathology, University of Alabama at Birmingham, Birmingham, AL, 35233, USA. Correspondence and requests for materials should be addressed to M.R. (email: mramani@peds.uab.edu) 
to hyperoxia from P2-14 indicated impairments in hippocampal protein synthesis and translation and predicted mitochondrial dysfunction ${ }^{10}$. Hyperoxic exposure can cause cell death ${ }^{11}$ and impair cell survival ${ }^{12}$ in the developing brain. Chronic OS due to $\mathrm{O}_{2}$ supplementation may negatively affect neuronal mitochondrial function and lead to neurodegenerative disorders ${ }^{13}$.

Area CA1, a region of the hippocampus crucial for the acquisition of long-term memory ${ }^{14-16}$, is highly vulnerable to $\mathrm{OS}^{17}$. Mitochondria isolated from CA1 neurons have been shown to generate more reactive oxygen species (ROS) than any other regions of the hippocampus ${ }^{18}$. Adequate mitochondrial function is essential for mechanisms required for learning and memory ${ }^{19}$ in the hippocampus. While mitochondrial dysfunction is associated with the pathogenesis of several neurodegenerative diseases in adults ${ }^{20}$, the impact of early-life mitochondrial dysfunction on long-term brain development and function is yet to be determined.

In this study, we hypothesized that prolonged hyperoxia exposure during the critical developmental period would permanently alter hippocampal mitochondrial function. Our objective was to determine the long-term changes in hippocampal mitochondrial respiratory complex protein expression and bioenergetic function in neonatal mice (P14) and young adult mice (14 weeks) exposed to hyperoxia from P2-P14.

\section{Results}

Targeted and global proteomics. Long-term effect of neonatal hyperoxia exposure on hippocampal mitochondrial complex I, II, and III protein expressions in young adult mice. Young adult mice exposed to hyperoxia as neonates had reduced amounts of complex I NADH Dehydrogenase [Ubiquinone] 1 Beta Subcomplex 8 (NDUFB8), and complex I NADH Dehydrogenase [Ubiquinone] 1 Beta Subcomplex 11 (NDUFB11) subunit proteins (Table 1). The levels of other detected complex I subunits and of complex II subunits were comparable between the groups (Table 1). Complex III cytochrome b-cl complex subunit 9 was increased in the hyperoxia-exposed group compared to air-exposed group (Table 1).

Long-term effect of neonatal hyperoxia exposure on hippocampal mitochondrial complex $I V$ and $V$ protein expressions in young adult mice. Young adult mice exposed to hyperoxia as neonates had less cytochrome $\mathrm{C}$ oxidase subunit 7B (COX7B) protein compared to air-exposed groups (Table 2). The amounts of other detected complex IV and $\mathrm{V}$ subunits were similar between the groups (Table 2).

Bioinformatic analysis of differentially expressed hippocampal proteins. Differentially expressed hippocampal proteins in young adult mice following neonatal hyperoxia exposure. With a cut-off of \pm 1.5 fold-change with P-value $<0.05$ (by analysis of variance), and a false discovery rate of $5 \%$, we identified a total of 196 hippocampal proteins that were differentially expressed in neonatal hyperoxia-exposed young adult mice compared to air-exposed young adult mice. Of these 196 proteins, 48 proteins were increased, and 148 were decreased following neonatal hyperoxia exposure. The heat map of the differentially expressed hippocampal proteins is shown in Supplemental Fig. S1. The full list of upregulated and downregulated proteins (limited to fold-change 1.5) in young adult mice exposed to neonatal hyperoxia are listed in Supplemental Tables S1 and S2, respectively. The top 10 differentially expressed hippocampal proteins in the hyperoxia-exposed young adult mice are listed in Table 3 . The protein classes that are upregulated and downregulated by hyperoxia exposure are shown in Fig. 1A,B, respectively.

Long-term effect of effect of neonatal hyperoxia exposure on hippocampal biological processes in young adult mice. Differentially expressed hippocampal proteins were predominantly involved in biological processes of cellular process, metabolic process, biogenesis, and protein localization (Fig. 1C,D). Among the upregulated hippocampal proteins, functions of $20(29.9 \%)$ proteins were associated with cellular process, $18(26.9 \%)$ were proteins associated with metabolic process, $9(13.4 \%)$ were proteins associated with the biogenesis process, and $6(9 \%)$ were proteins associated with the localization process (Fig. 1C). Among the downregulated proteins, functions of 62 $(27.8 \%)$ proteins were associated with the cellular process, $39(18.1 \%)$ were proteins associated with metabolic process, $26(12.1 \%)$ were proteins associated with biogenesis process, and 20 (9.6\%) were proteins associated to localization process (Fig. 1D).

Top canonical hippocampal pathways regulated by neonatal hyperoxia exposure in young adult mice. The top canonical pathways that were most impacted by neonatal hyperoxia exposure are listed in Table 4. Bioinformatic analysis of differentially expressed hippocampal proteins predicated that mitochondrial function $(\mathrm{P}=2.03 \mathrm{E}-06)$, oxidative phosphorylation $(\mathrm{P}=1.25 \mathrm{E}-05)$, GABA receptor signaling $(\mathrm{P}=1.26 \mathrm{E}-04)$, amyotrophic lateral sclerosis signaling $(\mathrm{P}=4.09 \mathrm{E}-04)$, and amyloid processing $(\mathrm{P}=5.34 \mathrm{E}-04)$ were impacted in young adult mice that had neonatal hyperoxia exposure. Fifteen proteins were associated with mitochondrial function, 11 were related to oxidative phosphorylation, 9 were related to GABA receptor signaling, 9 were proteins related to amyotrophic lateral sclerosis signaling, and 6 were proteins related to amyloid processing and were differentially expressed in the hyperoxia-exposed group.

Mitochondrial studies. Effects of neonatal hyperoxia exposure on hippocampal mitochondrial bioenergetics in neonatal mice (P14). Neonatal hyperoxia (exposure from P2-P14) exposure decreased both pyruvate/malate mitochondrial ATP linked $(\mathrm{P}=0.01)$ and complex I enzyme activity $(\mathrm{P}=0.01)$ at $\mathrm{P} 14$ (Fig. $2 \mathrm{~A}, \mathrm{D}$, respectively). No differences were observed between hyperoxia-exposed and air-exposed controls in ATP linked $\mathrm{O}_{2}$ consumption rates that utilized succinate (complex II substrate; $\mathrm{P}=0.73$ ) or complex IV activity $(\mathrm{P}=0.99)$ (Fig. 2C,E, respectively), consistent with the hypothesis that the observed differences were related to a complex I defect. Examination of state 4 minus basal $\mathrm{O}_{2}$ consumption rates also suggested increased oxygen consumption in the hyperoxia-exposed group $(\mathrm{P}=0.03)$, which could be linked to increased proton leak and/or oxidant generation 


\begin{tabular}{|c|c|c|}
\hline Molecule (Symbol) & $\begin{array}{l}\text { Protein } \\
\text { Log Fold Change in } \\
\text { Hyperoxia (vs. Air) }\end{array}$ & $\begin{array}{l}\text { P value for } \\
\text { protein change }\end{array}$ \\
\hline \multicolumn{3}{|l|}{ Complex I Protein Subunits } \\
\hline \multicolumn{3}{|c|}{ NADH Dehydrogenase [Ubiquinone] 1 Alpha Subcomplex } \\
\hline Subunit 2 (NDUFA2) & -0.27 & 0.81 \\
\hline Subunit 5 (NDUFA5) & +0.65 & 0.38 \\
\hline Subunit 6 (NDUFA6) & -0.18 & 0.90 \\
\hline Subunit 7 (NDUFA7) & +1.34 & 0.23 \\
\hline Subunit 8 (NDUFA8) & +2.19 & 0.08 \\
\hline Subunit 9 (NDUFA9) & +0.82 & 0.51 \\
\hline Subunit 10 (NDUFA10) & -0.54 & 0.65 \\
\hline Subunit 12 (NDUFA12) & +1.36 & 0.36 \\
\hline Subunit 13 (NDUFA13) & +1.29 & 0.26 \\
\hline Assembly Factor 3 (NDUFAF3) & -0.90 & 0.58 \\
\hline Assembly Factor 4 (NDUFAF4) & +0.26 & 0.85 \\
\hline Assembly Factor 5 (NDUFAF5) & +0.09 & 0.94 \\
\hline \multicolumn{3}{|c|}{ NADH Dehydrogenase [Ubiquinone] 1 Beta Subcomplex } \\
\hline Subunit 3 (NDUFB3) & +0.02 & 0.98 \\
\hline Subunit 4 (NDUFB4) & -0.35 & 0.79 \\
\hline Subunit 5 (NDUFB5) & +0.42 & 0.78 \\
\hline Subunit 7 (NDUFB7) & +1.10 & 0.46 \\
\hline Subunit 8 (NDUFB8) & -2.65 & 0.04 \\
\hline Subunit 10 (NDUFB10) & +0.37 & 0.63 \\
\hline Subunit 11, (NDUFB11) & -2.37 & 0.034 \\
\hline \multicolumn{3}{|l|}{ NADH Dehydrogenase [Ubiquinone] 1} \\
\hline Subunit C2 (NDUFC2) & +0.07 & 0.95 \\
\hline \multicolumn{3}{|l|}{ NADH Dehydrogenase [Ubiquinone] } \\
\hline Flavoprotein 1 (NDUFV1) & +1.94 & 0.17 \\
\hline Flavoprotein 2 (NDUFV2) & +0.54 & 0.19 \\
\hline \multicolumn{3}{|l|}{ Complex II Protein Subunits } \\
\hline \multicolumn{3}{|l|}{ Succinate dehydrogenase } \\
\hline Cytochrome b560 subunit (SDHC) & +0.89 & 0.53 \\
\hline Iron-sulfur subunit (SDHB) & +0.82 & 0.49 \\
\hline Flavoprotein subunit (SDHA) & -0.14 & 0.64 \\
\hline \multicolumn{3}{|l|}{ Complex III Protein Subunits } \\
\hline Cytochrome B5 Type B (CYB5B) & -0.38 & 0.78 \\
\hline Cytochrome b-c1 complex subunit 9 (UQCR10) & +4.44 & 0.0005 \\
\hline
\end{tabular}

Table 1. Long-term Effect of Neonatal Hyperoxia Exposure on Hippocampal Mitochondrial Complex I, II and III Proteins in Young Adult Mice ( $n=5$ in Air group, 6 in Hyperoxia group).

(Fig. 2B). Hyperoxia-exposed neonatal mice also had reduced citrate synthase activity $(\mathrm{P}=0.01)$ (Fig. 2F), compared to air-exposed neonatal mice.

Effects of neonatal hyperoxia exposure on hippocampal mitochondrial bioenergetics in young adult mice (14 weeks). Similar to the observations in neonates exposed to hyperoxia, adult mice (14 weeks old) that underwent neonatal hyperoxia exposure from P2-P14 had decreased mitochondrial ATP linked $\mathrm{O}_{2}$ consumption in the presence of complex I substrates (pyruvate/malate) $(\mathrm{P}=0.01)$ (Fig. 3A) whereas in the presence of succinate $(\mathrm{P}=0.74)$, no differences were observed relative to air-exposed controls (Fig. 3D). Similarly, no differences were observed in complex IV activity $(\mathrm{P}=0.45)$ between exposed and unexposed control groups (Fig. $3 \mathrm{G})$. Also, similar to the hyperoxia-exposed newborn mice, oligomycin induced state $4 \mathrm{O}_{2}$ consumption rates minus basal $\mathrm{O}_{2}$ consumption rates were increased $(\mathrm{P}=0.05)$ (Fig. $3 \mathrm{C})$, consistent with increased proton leak and/or oxidant generation. However, unlike in neonates, complex I activity $(\mathrm{P}=0.002)$ was significantly increased in the 14 -week old mice exposed to hyperoxia as neonates (Fig. 3E). Subgroup analysis by sex also showed that young adult male mice exposed to hyperoxia as neonates had decreased ATP linked $\mathrm{O}_{2}$ consumption (One Way ANOVA, mean difference 36.7, $\mathrm{P}=0.01$ ) (Fig. 3B) and increased complex I activity (One Way ANOVA, mean difference 13.66, $\mathrm{P}=0.03$ ) (Fig. $3 \mathrm{~F}$ ) compared to air-exposed young adult male mice. The difference in citrate synthase activity $(\mathrm{P}=0.78)$ seen in neonatal mice exposed to hyperoxia were no longer observed when assessed as young adults (Fig. 3H). 


\begin{tabular}{|c|c|c|}
\hline Molecule (Symbol) & $\begin{array}{l}\text { Protein } \\
\text { Log Fold Change in } \\
\text { Hyperoxia (vs. Air) }\end{array}$ & $\begin{array}{l}\text { P Value for } \\
\text { Protein Change }\end{array}$ \\
\hline \multicolumn{3}{|l|}{ Complex IV Protein Subunits } \\
\hline \multicolumn{3}{|l|}{ Cytochrome C Oxidase } \\
\hline Subunit 2 (COX2) & +0.67 & 0.06 \\
\hline Subunit 4 Isoform 1 (COX41) & +0.42 & 0.59 \\
\hline Subunit 5A (COX5A) & -1.42 & 0.33 \\
\hline Subunit 5B (COX5B) & +0.15 & 0.78 \\
\hline Subunit 6A1 (CX6A1) & +3.32 & 0.08 \\
\hline Subunit 6B1 (CX6B1) & +0.55 & 0.34 \\
\hline Subunit 6C (COX6C) & +0.19 & 0.87 \\
\hline Subunit 7A2 (CX7A2) & +0.96 & 0.51 \\
\hline Subunit 7B (COX7B) & -2.09 & 0.04 \\
\hline Subunit 7C (COX7C) & +0.39 & 0.39 \\
\hline Assembly Factor 7 (COA7) & +1.76 & 0.18 \\
\hline Subunit NDUFA4 (NDUA4) & +1.17 & 0.06 \\
\hline Translational Activator 1 (TACO1) & -0.74 & 0.58 \\
\hline \multicolumn{3}{|l|}{ Complex V Protein Subunits } \\
\hline \multicolumn{3}{|l|}{ ATP Synthase } \\
\hline Protein 8 (ATP8) & +0.66 & 0.25 \\
\hline Subunit Alpha (ATPA) & +0.16 & 0.34 \\
\hline Subunit Beta (ATPB) & +0.02 & 0.93 \\
\hline Subunit Delta (ATPD) & -0.34 & 0.78 \\
\hline Subunit Gamma (ATPG) & +0.44 & 0.48 \\
\hline Subunit F (ATPK) & -1.82 & 0.30 \\
\hline Subunit O (ATPO) & +0.47 & 0.23 \\
\hline Subunit D (ATP5H) & +0.73 & 0.06 \\
\hline Subunit G (ATP5L) & -0.33 & 0.85 \\
\hline Subunit E (ATP5I) & +0.89 & 0.11 \\
\hline Subunit Epsilon (ATP5E) & -1.35 & 0.36 \\
\hline Subunit S (ATP5S) & +0.88 & 0.37 \\
\hline
\end{tabular}

Table 2. Long-term Effect of Neonatal Hyperoxia Exposure on Hippocampal Mitochondrial Complex IV and V Proteins in Young Adult Mice ( $\mathrm{n}=5$ in Air group, 6 in Hyperoxia group).

Effects of neonatal hyperoxia exposure on hippocampal mitochondrial copy number in neonatal (P14) and young adult mice (14 weeks). No difference in the mitochondrial copy number was observed among the air- and hyperoxia-exposed neonatal mice (Fig. 4A). Similarly, no difference in the mitochondrial copy number was observed among air- and hyperoxia-exposed young adult mice (Fig. 4B).

\section{Discussion}

This is the first preclinical study to demonstrate the long-term adverse effect of early life hyperoxia on hippocampal mitochondrial function and mitochondrial respiratory chain protein expression. We discovered that hyperoxia exposure during a critical developmental period permanently impairs hippocampal mitochondrial function, alters the expression of specific respiratory chain subunits for complexes I and III, and impairs complex I activity in the hippocampus. As spatial memory deficits and other cognitive problems in the mouse model of bronchopulmonary dysplasia (BPD) correspond to the cognitive deficits seen in adolescents with BPD, these new observations suggest that permanent hippocampal mitochondrial dysfunction induced by early life oxygen exposure as a contributor to the pathophysiology of BPD associated cognitive dysfunction.

This study has several strengths. We have used unbiased proteomic analysis of whole hippocampal tissue using highly sensitive mass spectrometric methods. Rather than being limited to only mitochondrial proteins, our study also evaluated the long-term impact of early life oxygen exposure on all hippocampal proteins and used sophisticated bioinformatics analysis to define long-term changes in the hippocampal signaling pathways. This study also evaluated mitochondrial bioenergetics induced by hyperoxia exposure during the critical developmental period (P14) and young adult age (14 weeks, the age at which we observed cognitive dysfunction in our previous study). In addition to citrate synthase assay, a surrogate measure of mitochondrial content, we also measured mitochondrial copy number, an alternative measure for mitochondrial content.

There are also a few limitations to this study. The proteomics and the mitochondrial bioenergetic studies were performed on the whole hippocampus instead of specific hippocampal subfields which are known to play different roles in memory and learning. Furthermore, since proteomic and bioenergetic studies were done from the whole hippocampus, it is not possible to determine whether these early life oxygen-induced changes in hippocampal proteins and mitochondrial function were predominantly derived from neurons or glial cells or a 


\begin{tabular}{|c|c|c|}
\hline Protein (Symbol) & $\begin{array}{l}\text { Log Fold Change in } \\
\text { Hyperoxia Group }\end{array}$ & P value \\
\hline \multicolumn{3}{|l|}{ Up-regulated Proteins } \\
\hline Ras-related protein Rab-8A (RAB8A) & +4.78 & 0.032 \\
\hline Cytochrome b-c1 complex subunit 9 (UQCR9) & +4.44 & 0.0005 \\
\hline Regulator complex protein LAMTOR3 (LTOR3) & +4.35 & 0.02 \\
\hline Mitochondrial Ribosomal Protein L11 (MRPL11) & +4.19 & 0.003 \\
\hline Myelin proteolipid (PLP) & +4.05 & 0.003 \\
\hline \multicolumn{3}{|l|}{ Down-regulated Proteins } \\
\hline Filamin-C (FLNC) & -5.13 & $9.97 \mathrm{E}-07$ \\
\hline Vacuolar Protein Sorting-Associated Protein 52 Homolog (VPS52) & -4.74 & $1.79 \mathrm{E}-06$ \\
\hline Integrin Beta-1 (ITB1) & -4.50 & $5.77 \mathrm{E}-07$ \\
\hline Glucose-6-Phosphate 1-Dehydrogenase X (G6PD1) & -4.44 & 0.01 \\
\hline Teneurin-1 (TEN1) & -4.38 & 0.0001 \\
\hline
\end{tabular}

Table 3. Top 10 Differentially Expressed Hippocampal Proteins in Neonatal Hyperoxia-Exposed Young Adult Mice ( $\mathrm{n}=5$ in Air group, 6 in Hyperoxia group, $\mathrm{P}=$ Hyperoxia vs. Air group).

A

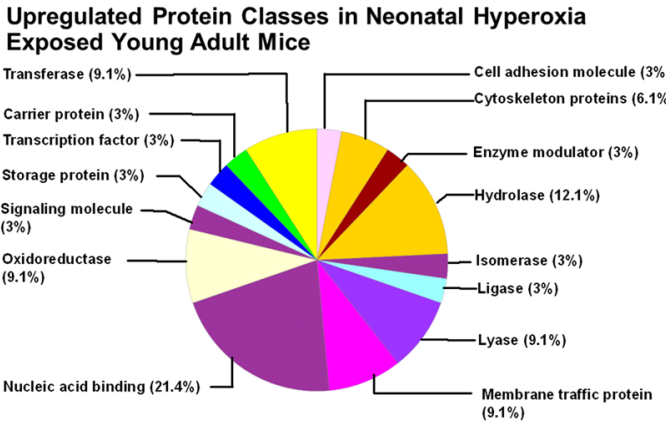

C

Upregulated Biological Processes in Neonatal Hyperoxia Exposed Young Adult Mice

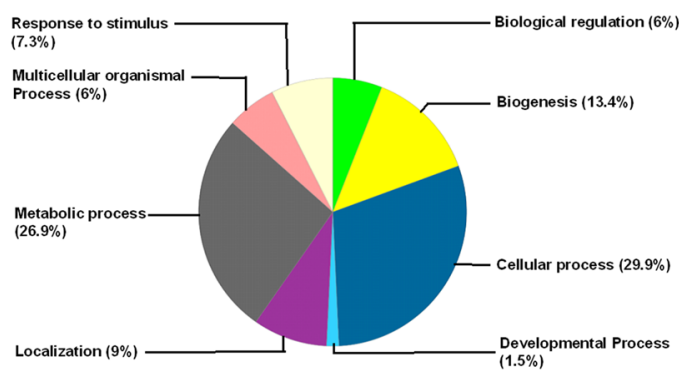

B

Downregulated Protein Classes in Neonatal Hyperoxia Exposed Youna Adult Mice

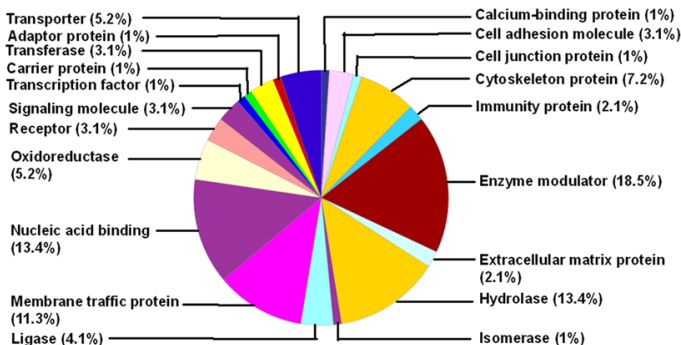

D

Downregulated Biological Processes in Neonatal Hyperoxia Exposed Young Adult Mice

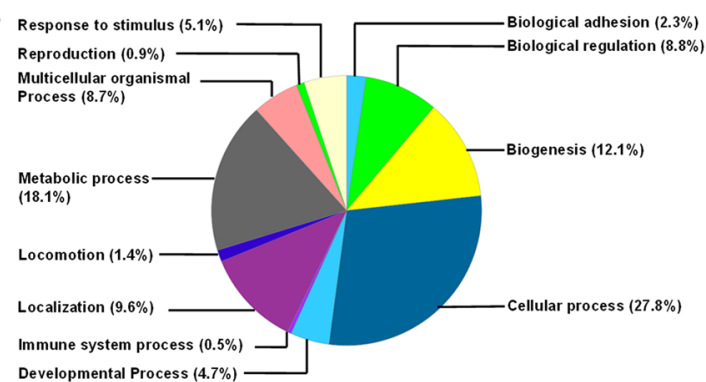

Figure 1. Distribution of hippocampal proteins in young adult mice exposed to neonatal hyperoxia. (A) Graphical representation of the distribution of upregulated hippocampal proteins by class in the hyperoxiaexposed group. (B) Graphical representation of the distribution of downregulated hippocampal proteins by class in the hyperoxia-exposed group. (C) Graphical representation of the distribution of upregulated hippocampal proteins by biological processes in the hyperoxia-exposed group. (D) Graphical representation of the distribution of downregulated hippocampal proteins by biological processes in the hyperoxia-exposed group.

combination of both. In addition, proteomic methods often require large sample sizes, as there is often much variation from one sample to another, and even large differences may not end up being statistically significant. In this study, we focused on proteomics and mitochondrial bioenergetics only from the hippocampal homogenates, and not from other regions of the brain such as cerebellum, amygdala, corpus callosum, and white matter tracts which might also have impacted by hyperoxia exposur ${ }^{21-23}$. Even though hippocampal complexes I and IV activities were measured, complex III and complex V activities were not measured due to technical difficulties and the size of the hippocampus.

Though lung and brain development in newborn mouse pups corresponds to 24-28 weeks of gestation in human preterm infants, the highly efficient redox and gas exchange system of the C57BL6 mice ${ }^{24}$ requires supraphysiological concentrations $\left(85 \% \mathrm{O}_{2}\right)$ and a longer duration (P2-14) of oxygen exposure to induce human $\mathrm{BPD}$-like lung pathology $\mathrm{y}^{9,25}$. Our model, while not an exact simulation of the human preterm infant in the 


\begin{tabular}{|l|l|l|}
\hline & P-Value & $\begin{array}{l}\text { Overlap } \\
\text { (percentage; number of proteins } \\
\text { differentially expressed/number } \\
\text { of proteins in pathway) }\end{array}$ \\
\hline Mitochondrial dysfunction & $2.03 \mathrm{E}-06$ & $8.8 ; 15 / 171$ \\
\hline Oxidative phosphorylation & $1.25 \mathrm{E}-05$ & $10.1 ; 11 / 109$ \\
\hline GABA receptor signaling & $1.26 \mathrm{E}-04$ & $9.5 ; 9 / 95$ \\
\hline Amyotrophic lateral sclerosis signaling & $4.09 \mathrm{E}-04$ & $8.1 ; 9 / 111$ \\
\hline Amyloid processing & $5.34 \mathrm{E}-04$ & $11.8 ; 6 / 51$ \\
\hline
\end{tabular}

Table 4. Top Canonical Pathways Involved in Neonatal Hyperoxia-Exposed Young Adult Mice by Ingenuity Pathway Analysis (Using Proteomics Data, $n=5$ in Air group, 6 in Hyperoxia group, $\mathrm{P}=$ Hyperoxia vs. Air group).

neonatal intensive care unit, reproduces both structurally the hippocampal shrinkage and functionally the associated memory deficits ${ }^{26}$ seen in adolescents and young adults with BPD.

The hippocampus, a region of the brain that plays a vital role in consolidating short memory into long-term memory ${ }^{14-16}$, is highly vulnerable to oxidative stress ${ }^{17}$. Oxygen exposure causes neuronal cell death in developing brain ${ }^{11,12}$, and prolonged oxidative stress impairs neuronal mitochondrial function ${ }^{13}$. Neurons in the hippocampus are critically dependent on their mitochondrial function for the strengthening of synapses, a cellular response responsible for the formation and maintenance of long-term memory ${ }^{27-29}$. In neurons, mitochondria generate about $90 \%$ of the ATP by oxidative phosphorylation. In oxidative phosphorylation, oxygen is the terminal electron acceptor of the mitochondrial electron transport chain (ETC). ETC transfers electrons from high energy metabolites through a series of electron acceptors (carriers) to drive the generation of ATP from ADP ${ }^{30}$. The redox state of the respiratory chain is governed by the trans-membrane proton gradient and the membrane potential $^{31}$. The redox energy used for ATP generation also leads to the production ROS ${ }^{32}$. Excessive ROS production following hyperoxia exposure can potentially overwhelm antioxidant defense mechanisms and leads to mitochondrial damage ${ }^{33,34}$ and cellular death ${ }^{35}$.

Our mitochondrial functional assessments show that early life hyperoxia exposure not only reduces ATP linked oxygen consumption in the hippocampus in the neonatal period (P14) but also in the young adult (14 weeks). In addition, our study also shows a persistent increase in the rate of oxygen consumption at state 4 respiration, (a surrogate measure of proton leak) in young adults exposed to hyperoxia as neonates, and suggests uncoupling between substrate oxidation and ATP synthesis. Alterations in mitochondrial coupling can alter ROS production ${ }^{36-38}$ and ATP synthesis ${ }^{39}$. Though the amount of ATP produced by the hippocampal tissue was not measured in this study, the decrease in ATP linked oxygen consumption and increase in state 4 proton leak both at P14 and 14 weeks suggest that early life oxygen exposure permanently impairs mitochondrial efficiency in the generation of ATP. The neonatal hyperoxia-induced hippocampal mitochondrial dysfunction measured through bioenergetic studies in young adult mice is consistent with the mitochondrial dysfunction predicted through proteomic analysis.

Complex I (NADH: ubiquinone oxidoreductase), the first and largest enzyme in the ETC, has been consistently shown to be vulnerable to oxidative stress-mediated dysfunction ${ }^{40}$. It is also thought to be the main site of ROS production ${ }^{41,42}$, and its impairment leads to an increase in ROS production ${ }^{43}$. Decreased complex I activity seen in hyperoxia-exposed neonatal mice suggests that oxygen exposure either directly or indirectly impairs complex I function. At 14 weeks, the targeted hippocampal proteomic analysis determined decreases in complex I NDUFB8 and NDUFB11 subunits in neonatal hyperoxia-exposed mice, inner membrane subunits that are located in the membrane arm of complex I together along with proton pumping subunits. While neither of these subunits is thought to be directly involved in catalysis, decreased levels of NDUFB8 associated with AD in rodent model ${ }^{44}$. However, mitochondrial bioenergetic studies indicated an increase in complex I activity at 14 weeks. The persistent decreased ATP linked $\mathrm{O}_{2}$ consumption and increased state 4 proton leak at 14 weeks despite the increase in complex I activity in the young adult mice exposed to hyperoxia suggest persistent mitochondrial dysfunction and inadequate compensation by the later increase in complex I activity following hyperoxia-induced decreases in the newborn. Neonatal hyperoxia did not affect complex IV activity either at P14 or 14 weeks suggesting that either the complex IV enzyme is not as highly vulnerable to oxidative stress as complex I or it is well adapted to oxidative stress-induced injury. Though changes in hyperoxia-induced complex III and V activity are possible, comparable succinate-induced oxygen consumption between hyperoxia and air-exposed neonatal and young adult mice indicate that dysfunction in oxygen consumption noted with hyperoxia exposure is mainly induced by alterations in complex I function.

Mitochondrial metabolism and signaling pathways that regulate cell death are sexually dimorphic ${ }^{45}$. Compared to the female, the male hippocampus has a lower level of endogenous antioxidant defense systems ${ }^{46}$ and produces more $\mathrm{ROS}^{47}$. We determined that hyperoxia-exposed young males had reduced ATP linked $\mathrm{O}_{2}$ consumption and increased complex I activity compared to hyperoxia-exposed young females. These observations are clinically important because prematurity associated neurodevelopmental outcomes ${ }^{48}$ and neurodevelopmental disorders (e.g., Autism) ${ }^{49}$ preferentially affects male sex. Citrate synthase, a surrogate marker for mitochondrial volume ${ }^{50}$, was reduced by hyperoxia in neonates (P14) and normalized in young adults (14 weeks). However, when we independently verified the citrate synthase (mitochondrial content) results with mitochondrial copy number by qPCR, we did not observe any differences in mitochondrial copy number among the groups both either in neonatal or young adult mice. This suggests that even though early life hyperoxia exposure permanently impairs hippocampal mitochondrial function, it may not have significant short-term or long-term impact on hippocampal mitochondrial biogenesis. 
A

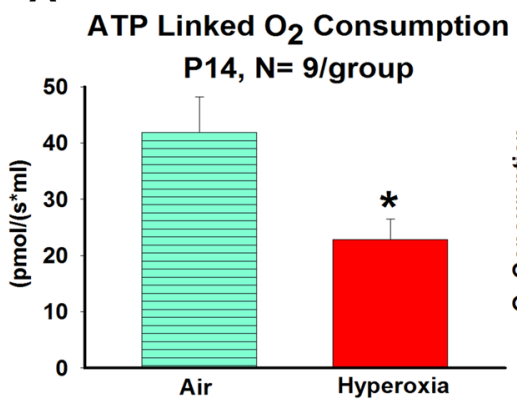

D

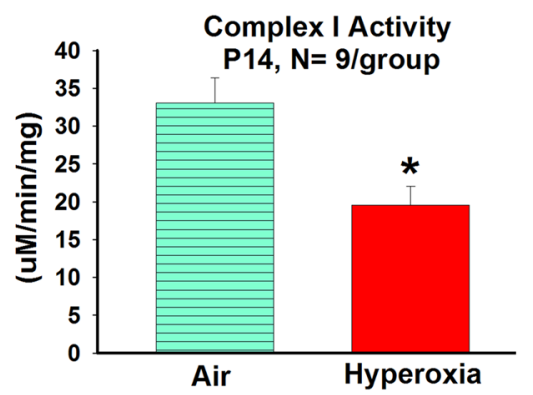

B State 4 Respiration Proton Leak P14, N= 9/group

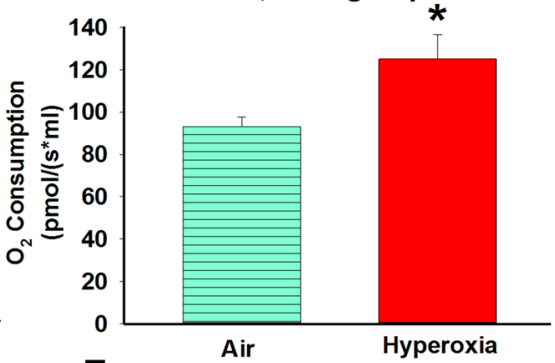

E

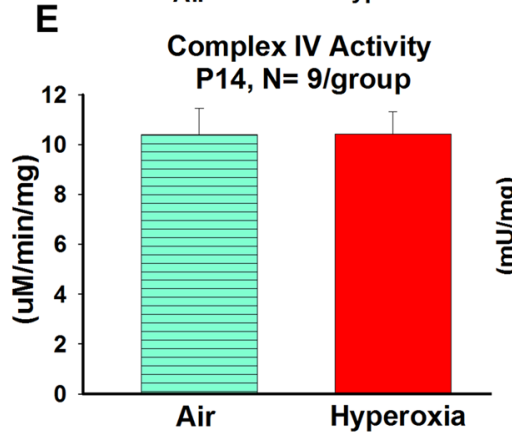

C

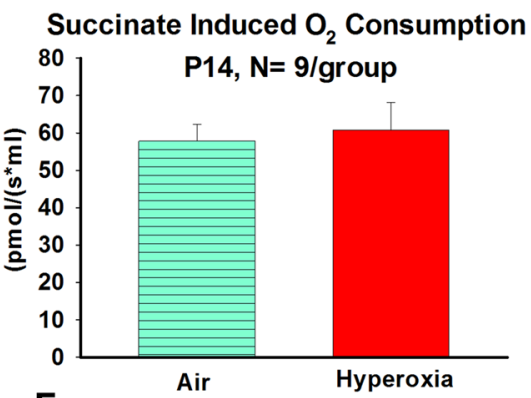

F

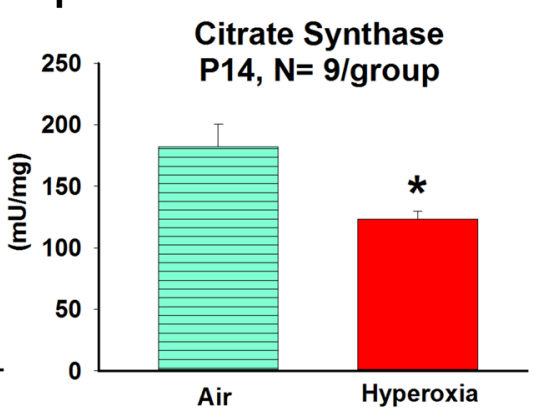

Figure 2. Effects of neonatal hyperoxia exposure on hippocampal mitochondrial bioenergetics in neonatal mice. $\mathbf{A}=$ ATP linked oxygen consumption, $\mathbf{B}=$ State 4 respiration proton leak, $\mathbf{C}=$ Succinate induced oxygen consumption, $\mathbf{D}=$ Complex I activity measured by assay, $\mathbf{E}=$ Complex IV activity measured by assay, and $\mathbf{F}=$ Citrate synthase activity measured by assay. Air-exposed: cyan bars with horizontal stripes and hyperoxiaexposed: solid red bars; means \pm SEM; $n=9$ in air and 9 in hyperoxia. $* \mathrm{p}<0.05$ vs. air-exposed mice.
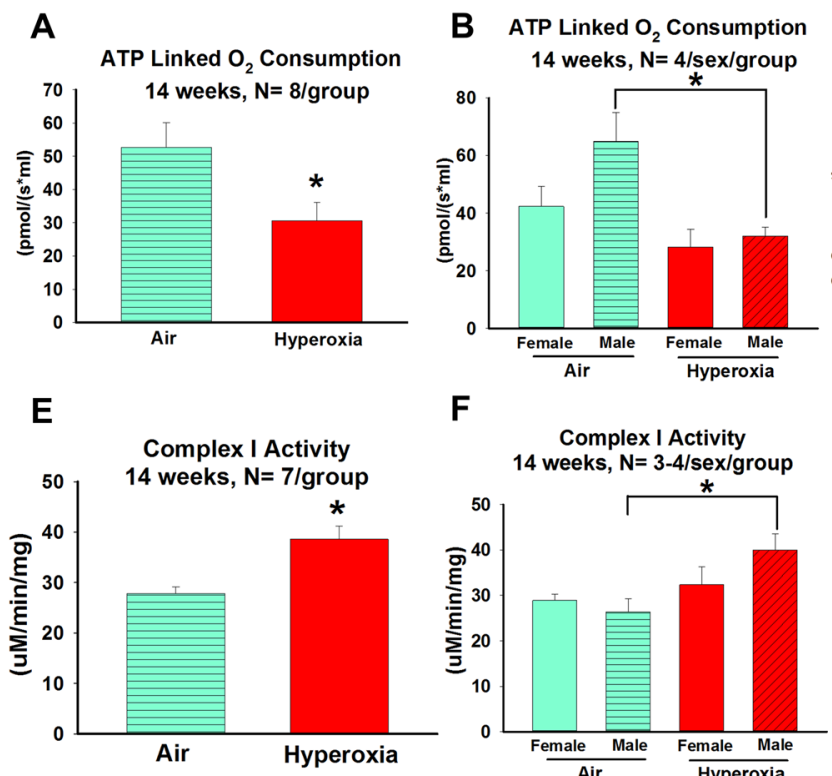

$\mathbf{F}$

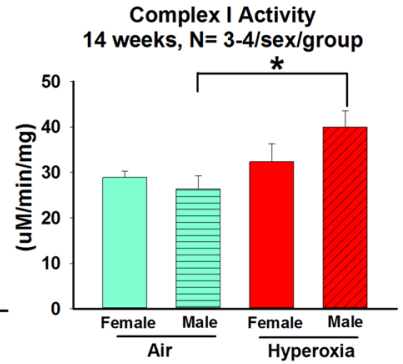

C State 4 Respiration Proton Leak

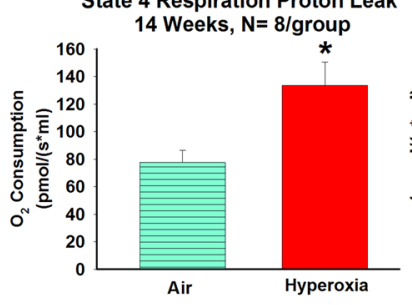

Succinate Induced $\mathrm{O}_{2}$ Consumption

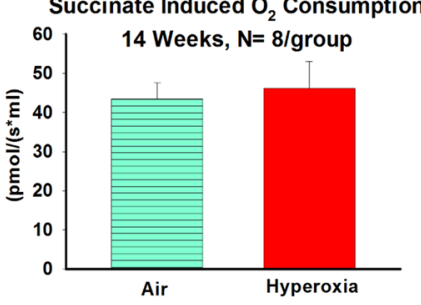

H

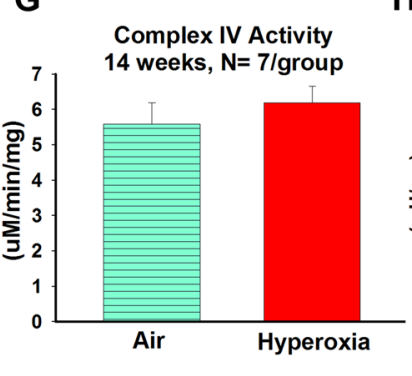

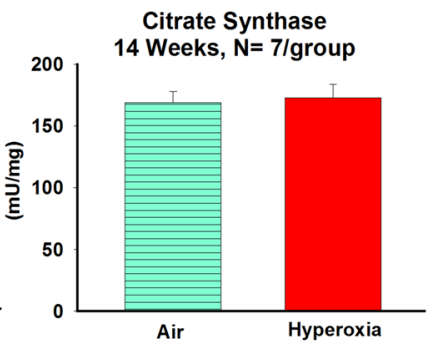

Figure 3. Effects of neonatal hyperoxia exposure on hippocampal mitochondrial bioenergetics in young adult mice. $\mathbf{A}=$ ATP linked oxygen consumption, $\mathbf{B}=$ ATP linked oxygen consumption by sex, $\mathbf{C}=$ State 4 respiration proton leak, $\mathbf{D}=$ Succinate induced oxygen consumption, $\mathbf{E}=$ Complex I activity measured by assay, $\mathbf{F}=$ Complex I activity by Sex, $\mathbf{G}=$ Complex IV activity measured by assay, and $\mathbf{H}=$ Citrate synthase activity measured by assay. (A,C,D,E,G and G); air-exposed: cyan bars with horizontal stripes and hyperoxia-exposed: solid red bars; means \pm SEM; $\mathrm{n}=7-8$ in air and 7-9 in hyperoxia. * $\mathrm{p}<0.05$ vs. air-exposed mice. (B,F); Airexposed females: solid cyan bars, air-exposed males: cyan bars with horizontal stripes, hyperoxia-exposed females: solid red bar, and hyperoxia-exposed males, red bars with angled stripes; means \pm SEM; $n=4 / \operatorname{sex} /$ group. ${ }^{*} \mathrm{p}<0.05=$ air-exposed mice vs. hyperoxia-exposed males by One Way ANOVA. 

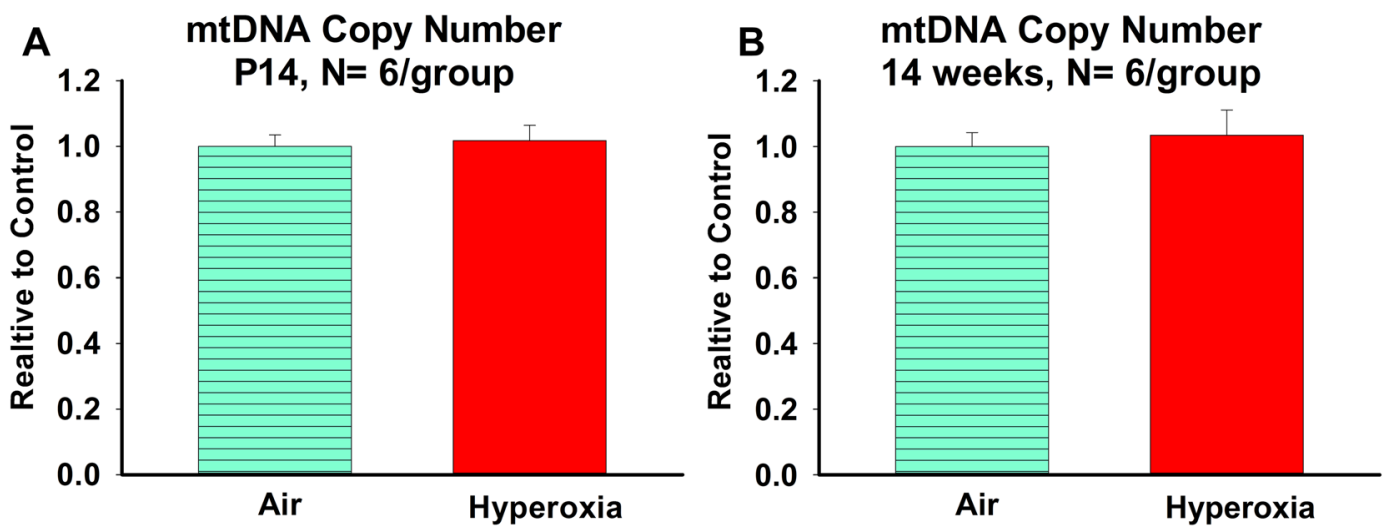

Figure 4. Effects of neonatal hyperoxia exposure on hippocampal mitochondrial DNA copy number in neonatal and young adult mice. $\mathbf{A}=$ Mitochondrial copy number relative to air exposed controls in neonatal (P14) mice and $\mathbf{B}=$ Mitochondrial copy number relative to air exposed controls in young adult (P14) mice. Airexposed: cyan bars with horizontal stripes and hyperoxia-exposed: solid red bars; means \pm SEM; $n=6$ in air and 6 in hyperoxia. $* \mathrm{p}<0.05$ vs. air-exposed mice.

In addition, hyperoxia-induced changes in the expression of Ras-related protein Rab-8A (involved in vesicular trafficking and neurotransmitter), Teneurin-1 (increases hippocampal dendritic arborization and spine density) and their roles in hyperoxia-induced cognitive dysfunction need further investigation. Also, reduction in hippocampal glucose-6-phosphate 1-dehydrogenase X (G6PD1) level in hyperoxia-exposed young adult mice suggests that early life hyperoxia exposure permanently impairs cytosolic oxidative phosphorylation, a process that is critical for the NADPH production. Since adequate NADPH level is essential for cellular oxidative stress regulation $^{51}$, it is possible that impaired hippocampal oxidative phosphorylation in hyperoxia-exposed young adult mice could have also impaired its ability to defend against oxidative stress even under normal ambient air conditions.

Our data also indicate that aberrant GABAergic signaling ${ }^{52}$ and amyloid processing are associated with cognitive deficits, and these pathways have been linked to neurodegenerative conditions ${ }^{53}$. Additional studies are needed to evaluate the contribution of these canonical pathways to impaired memory and hippocampal dysfunction induced by oxidative stress and to define how they interact with mitochondrial dysfunction. Since mitochondrial biogenesis is not impacted by early hyperoxia, we speculate that early oxidative stress possibly alterted complex I protein structure leading to an increase in mitochondrial ROS production which in turn may contribute to oxidative damage to mitochondrial DNA, altered mitophagy, and mitochondrial structure leading to long-term changes in complex I function and overall mitochondrial function (Supplemental Fig. S2). It is also possible that neonatal hyperoxia-induced phenotype might have originated not only from the initial insult (direct oxidative stress) to the complex I and other ETC enzymes but also due to the signaling pathways such as mitochondrial UQCR9, MRPL11 RAB8A, G6PD, and Teneurin-1 that are poorly compensated at a later point.

\section{Conclusion}

This study demonstrated that supraphysiological oxygen exposure during a critical period in neonatal development has a permanent negative impact on hippocampal mitochondria. The pathophysiology of neonatal hyperoxia-induced permanent mitochondrial dysfunction is complex. Future studies designed to quantitate mitochondrial DNA damage, ATP, and ROS levels are needed to determine the mechanisms by which early hippocampal complex I dysfunction induces permanent complex I dysfunction and the development of spatial memory deficits.

\section{Materials and Methods}

All protocols were approved by the UAB Institutional Animal Care and Use Committee (IACUC) and were consistent with the PHS Policy on Humane Care and Use of Laboratory Animals (Office of Laboratory Animal Welfare, Aug 2002) and the Guide for the Care and Use of Laboratory Animals (National Research Council, National Academy Press, 1996).

Animal model. C57BL/6J dams and their pups of both sexes were exposed to either normobaric hyperoxia $\left(85 \% \mathrm{O}_{2}, \mathrm{~N}=6\right)$ or normobaric $21 \% \mathrm{O}_{2}$ ambient air (Air, $\left.\mathrm{N}=6\right)$ from the second postnatal day (P2) until postnatal day 14 (P14), returned to room air, and maintained on standard rodent diet and light/dark cycling in microisolator cages until 14 weeks of age (Fig. 5A) ${ }^{25}$. An additional set of mice were exposed to either $85 \% \mathrm{O}_{2}$ (Hyperoxia, $\mathrm{N}=6$ ) or $21 \% \mathrm{O}_{2}$ (Air, $\mathrm{N}=6$ ) and sacrificed at P14 (Fig. $5 \mathrm{~A}$ ).

At 14 weeks, hippocampal proteins were analyzed using unbiased proteomic profiling using mass spectrometry. Initially, the targeted protein analysis was performed for hippocampal mitochondrial respiratory complex proteins. Subsequently, bioinformatics analysis was conducted on all other differentially expressed hippocampal proteins between hyperoxia and air-exposed groups. At P14 and 14 weeks of age, hippocampal tissues were analyzed for mitochondrial bioenergetic functions, complex I, IV, citrate synthase activity, and mitochondrial copy number. 


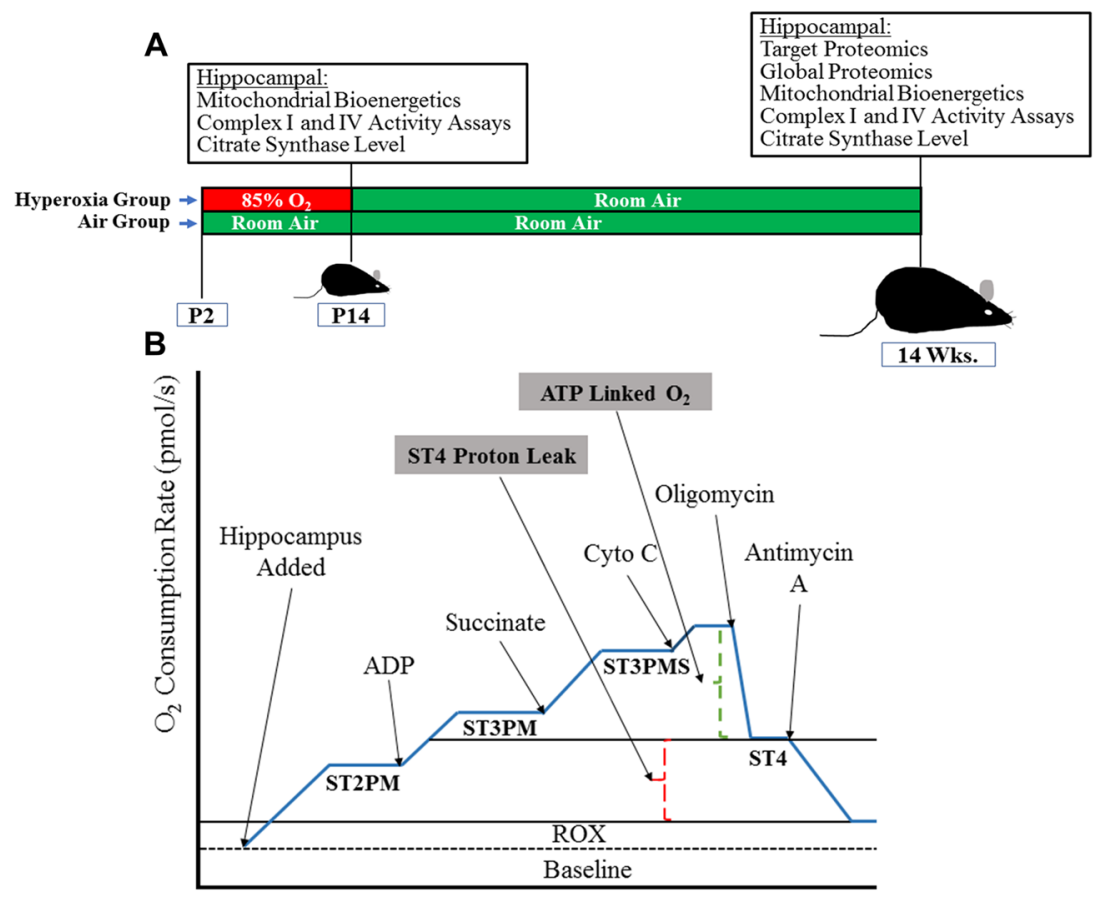

Figure 5. Schematics of the animal model and mitochondrial respiratory protocol. (A) Represents schematic of hyperoxia exposure from P2-14 and experimental studies done at P14 and 14 weeks. (B) Represents schematic of the mitochondrial respiratory protocol used in the high-resolution respirometry with sequentially added substrates and the calculations to assess the mitochondrial bioenergetic function using whole hippocampal tissue. $\mathrm{ST} 2 \mathrm{PM}=$ State 2 respiration with pyruvate and malate, ST3PM $=$ State 3 respiration with pyruvate and malate, $\mathrm{ADP}=$ Adenosine diphosphate, $\mathrm{ST} 3 \mathrm{PMS}=$ State 3 respiration with pyruvate, malate and succinate, $\mathrm{ST} 4=$ State 4 respiration following oligomycin, ATP Linked $\mathrm{O}_{2}=$ Adenosine triphosphate linked $\mathrm{O}_{2}$ consumption, $\mathrm{ST} 4$ Proton Leak $=$ State 4 respiration proton leak, $\mathrm{ROX}=$ Residual $\mathrm{O}_{2}$ consumption, and Baseline $=$ Baseline $\mathrm{O}_{2}$ consumption.

Mass spectrometry. At 14 weeks, following cervical dislocation, the whole brain was harvested from the mice, and hippocampi were removed in a sterile manner ${ }^{10}$. Then, Tissue was homogenized using Qiagen tissue lyser (Qiagen, MD, USA) in T-PER + Halt protease inhibitors + PMSF solution, and protein assay was performed using BCA protein assay kit (Thermo Fisher Scientific, MA, USA) ${ }^{54}$. The mass spectrometric analysis of hippocampal proteins was performed as previously described ${ }^{10}$.

Proteomics data assessment. Differentially expressed proteins (fold change \pm 1.5 fold and $\mathrm{p}<0.05$ ) were identified using T-test and further analyzed. As previously done ${ }^{10}$, functional analysis was performed using PANTHER (Protein ANalysis THrough Evolutionary Relationships) ${ }^{55}$ and Ingenuity Pathway Analysis (QIAGEN Inc. MD, USA). Heat maps were generated using pheatmap package V.1.0.7 in R program.

Mitochondrial bioenergetic studies (high-resolution respiratory). Whole hippocampus (right) was harvested and placed in ice cold artificial cerebrospinal fluid that contained glucose, BSA, EGTA, pyruvate, and mitochondrial respiration buffer, as previously described ${ }^{56}$. Briefly, hippocampal tissue was permeabilized with saponin $(5 \mathrm{mg} / \mathrm{mL}, 30$ minutes) and high-resolution respirometry performed using a two-channel respirometer (Oroboros Oxygraph-2k with DatLab software; Oroboros, Innsbruck, Austria). Reactions were conducted at $37^{\circ} \mathrm{C}$ in a $2 \mathrm{ml}$ chamber containing air-saturated mitochondrial respiration buffer (MiR03) under continuous stirring.

As illustrated in Fig. $4 \mathrm{~B}, \mathrm{O}_{2}$ consumption rates were measured in the presence of substrates $(5 \mathrm{mM}$ malate, $15 \mathrm{mM}$ pyruvate, $2.5 \mathrm{mM}$ ADP, $10 \mathrm{mM}$ succinate), and inhibitors $(0.5 \mu \mathrm{M}$ oligomycin, 5 um antimycin A) to assess state 2 (substrate alone), state 3 (substrate + ADP) and oligomycin induced state 4 respiration rates. Non-mitochondrial oxygen consumption was determined in the presence of antimycin A. Adenosine triphosphate (ATP) linked $\mathrm{O}_{2}$ consumption rate was determined by State 3 (substrates + ADP) - State 4 (oligomycin) = ATP linked rate. Non-ATP linked $\mathrm{O}_{2}$ consumption rate was determined by State 4 (oligomycin) non-mitochondrial oxygen consumption (antimycin A). Potential differences in $\mathrm{O}_{2}$ consumption rates based upon substrate utilization at complex I or II were assessed using pyruvate/malate or succinate, respectively, in the presence of ADP.

Complex I, IV and citrate synthase activity assays. Complex I, IV, and citrate synthase activities were measured from hippocampal (left) homogenates as previously described ${ }^{57-59}$; complex I activities were measured from freshly extracted tissues. 
Mitochondrial DNA copy number (qPCR). Mitochondrial DNA copy number was determined by QPCR as previously described ${ }^{60}$. Briefly, DNA was extracted from hippocampus homogenates from neonatal (P14) and young adult (14 weeks) mice exposed to room air or hypoxia from P2-14 using a Qiagen DNA ${ }^{\circledR}$ Mini Kit (Qiagen). DNA was quantified via fluorescence using Quant-iT ${ }^{\mathrm{TM}}$ PicoGreen ${ }^{\mathrm{TM}}$ dsDNA Assay Kit (Invitrogen). $15 \mathrm{ng}$ of DNA from each sample was used for quantitative PCR (qPCR). PCR products underwent electrophoresis for 2 hours at 90 volts on $10 \%$ polyacrylamide gels. Gels were stained with ethidium bromide for 45 minutes and imaged on an Amersham ${ }^{\mathrm{TM}}$ Imager 600 (GE Healthcare). All samples were loaded in duplicate and mitochondrial DNA copy number was quantified by measuring band intensities for each age group relative to age-matched room air-exposed controls using ImageQuant (GE Healthcare).

Statistical analysis. Results were expressed as means \pm SE. Multiple comparisons testing (Student-NewmanKeuls) was performed if statistical significance $(p<0.05)$ was noted by ANOVA.

\section{References}

1. Hauspurg, A. K. et al. Blood gases and retinopathy of prematurity: the ELGAN Study. Neonatology 99, 104-111, https://doi. org/10.1159/000308454 (2011).

2. SUPPORT Study Group of the Eunice Kennedy Shriver NICHD Neonatal Research Network et al. Target ranges of oxygen saturation in extremely preterm infants. N Engl J Med 362, 1959-1969, https://doi.org/10.1056/NEJMoa0911781 (2010).

3. Fawke, J. et al. Lung function and respiratory symptoms at 11 years in children born extremely preterm: the EPICure study. American journal of respiratory and critical care medicine 182, 237-245, https://doi.org/10.1164/rccm.200912-1806OC (2010).

4. Skromme, K., Vollsaeter, M., Oymar, K., Markestad, T. \& Halvorsen, T. Respiratory morbidity through the first decade of life in a national cohort of children born extremely preterm. BMC pediatrics 18, 102, https://doi.org/10.1186/s12887-018-1045-7 (2018).

5. Hintz, S. R. et al. Early-childhood neurodevelopmental outcomes are not improving for infants born at $<25$ weeks' gestational age. Pediatrics 127, 62-70, https://doi.org/10.1542/peds.2010-1150 (2011).

6. Schmidt, B. et al. Impact of bronchopulmonary dysplasia, brain injury, and severe retinopathy on the outcome of extremely lowbirth-weight infants at 18 months: results from the trial of indomethacin prophylaxis in preterms. JAMA: the journal of the American Medical Association 289, 1124-1129 (2003).

7. Hintz, S. R. et al. Changes in neurodevelopmental outcomes at 18 to 22 months' corrected age among infants of less than 25 weeks' gestational age born in 1993-1999. Pediatrics 115, 1645-1651, https://doi.org/10.1542/peds.2004-2215 (2005).

8. Trittmann, J. K., Nelin, L. D. \& Klebanoff, M. A. Bronchopulmonary dysplasia and neurodevelopmental outcome in extremely preterm neonates. European journal of pediatrics 172, 1173-1180, https://doi.org/10.1007/s00431-013-2016-5 (2013).

9. Ramani, M., van Groen, T., Kadish, I., Bulger, A. \& Ambalavanan, N. Neurodevelopmental impairment following neonatal hyperoxia in the mouse. Neurobiology of disease 50, 69-75, https://doi.org/10.1016/j.nbd.2012.10.005 (2013).

10. Ramani, M. et al. Supraphysiological Levels of Oxygen Exposure During the Neonatal Period Impairs Signaling Pathways Required for Learning and Memory. Sci Rep 8, 9914, https://doi.org/10.1038/s41598-018-28220-4 (2018).

11. Felderhoff-Mueser, U. et al. Oxygen causes cell death in the developing brain. Neurobiology of disease 17, 273-282, https://doi. org/10.1016/j.nbd.2004.07.019(2004).

12. Sengoku, T., Murray, K. M. \& Wilson, M. E. Neonatal hyperoxia induces alterations in neurotrophin gene expression. Int J Dev Neurosci 48, 31-37, https://doi.org/10.1016/j.ijdevneu.2015.11.003 (2016).

13. Guo, C., Sun, L., Chen, X. \& Zhang, D. Oxidative stress, mitochondrial damage and neurodegenerative diseases. Neural Regen Res $\mathbf{8}$, 2003-2014, https://doi.org/10.3969/j.issn.1673-5374.2013.21.009 (2013).

14. Stevenson, R. F. et al. Hippocampal CAl gamma power predicts the precision of spatial memory judgments. Proceedings of the National Academy of Sciences of the United States of America 115, 10148-10153, https://doi.org/10.1073/pnas.1805724115 (2018).

15. Squire, L. R. Memory and the hippocampus: a synthesis from findings with rats, monkeys, and humans. Psychological review 99 , 195-231 (1992).

16. Tsien, J. Z., Huerta, P. T. \& Tonegawa, S. The essential role of hippocampal CA1 NMDA receptor-dependent synaptic plasticity in spatial memory. Cell 87, 1327-1338 (1996).

17. Wang, X. et al. Genome-wide transcriptome profiling of region-specific vulnerability to oxidative stress in the hippocampus. Genomics 90, 201-212, https://doi.org/10.1016/j.ygeno.2007.03.007 (2007).

18. Mattiasson, G., Friberg, H., Hansson, M., Elmer, E. \& Wieloch, T. Flow cytometric analysis of mitochondria from CA1 and CA3 regions of rat hippocampus reveals differences in permeability transition pore activation. Journal of neurochemistry $\mathbf{8 7}, 532-544$ (2003).

19. Levy, M., Faas, G. C., Saggau, P., Craigen, W. J. \& Sweatt, J. D. Mitochondrial regulation of synaptic plasticity in the hippocampus. The Journal of biological chemistry 278, 17727-17734, https://doi.org/10.1074/jbc.M212878200 (2003).

20. Yan, M. H., Wang, X. \& Zhu, X. Mitochondrial defects and oxidative stress in Alzheimer disease and Parkinson disease. Free Radic Biol Med 62, 90-101, https://doi.org/10.1016/j.freeradbiomed.2012.11.014 (2013).

21. Scheuer, T. et al. Oligodendroglial maldevelopment in the cerebellum after postnatal hyperoxia and its prevention by minocycline. Glia 63, 1825-1839, https://doi.org/10.1002/glia.22847 (2015).

22. Scheuer, T. et al. Neonatal Hyperoxia Perturbs Neuronal Development in the Cerebellum. Mol Neurobiol, https://doi.org/10.1007/ s12035-017-0612-5 (2017).

23. Du, M. et al. Effects of the Notch signalling pathway on hyperoxia-induced immature brain damage in newborn mice. Neuroscience letters 653, 220-227, https://doi.org/10.1016/j.neulet.2017.05.065 (2017).

24. Rogers, L. K., Tipple, T. E., Nelin, L. D. \& Welty, S. E. Differential responses in the lungs of newborn mouse pups exposed to $85 \%$ or $>95 \%$ oxygen. Pediatric research 65,33-38, https://doi.org/10.1203/PDR.0b013e31818a1d0a (2009).

25. Ramani, M., van Groen, T., Kadish, I., Ambalavanan, N. \& McMahon, L. L. Vitamin A and retinoic acid combination attenuates neonatal hyperoxia-induced neurobehavioral impairment in adult mice. Neurobiol Learn Mem 141, 209-216, https://doi. org/10.1016/j.nlm.2017.04.013 (2017).

26. Gimenez, M. et al. Hippocampal gray matter reduction associates with memory deficits in adolescents with history of prematurity. NeuroImage 23, 869-877, https://doi.org/10.1016/j.neuroimage.2004.07.029 (2004).

27. Misko, A., Jiang, S., Wegorzewska, I., Milbrandt, J. \& Baloh, R. H. Mitofusin 2 is necessary for transport of axonal mitochondria and interacts with the Miro/Milton complex. The Journal of neuroscience: the official journal of the Society for Neuroscience 30, 4232-4240, https://doi.org/10.1523/JNEUROSCI.6248-09.2010 (2010).

28. Mironov, S. L. \& Symonchuk, N. ER vesicles and mitochondria move and communicate at synapses. J Cell Sci 119, 4926-4934, https://doi.org/10.1242/jcs.03254 (2006).

29. Murthy, V. N. \& De Camilli, P. Cell biology of the presynaptic terminal. Annual review of neuroscience 26, 701-728, https://doi. org/10.1146/annurev.neuro.26.041002.131445 (2003).

30. Magistretti, P. J. \& Allaman, I. A cellular perspective on brain energy metabolism and functional imaging. Neuron 86, 883-901, https://doi.org/10.1016/j.neuron.2015.03.035 (2015). 
31. Lambert, A. J. \& Brand, M. D. Superoxide production by NADH:ubiquinone oxidoreductase (complex I) depends on the $\mathrm{pH}$ gradient across the mitochondrial inner membrane. Biochem J 382, 511-517, https://doi.org/10.1042/BJ20040485 (2004).

32. Jensen, P. K. Antimycin-insensitive oxidation of succinate and reduced nicotinamide-adenine dinucleotide in electron-transport particles. II. Steroid effects. Biochim Biophys Acta 122, 167-174 (1966).

33. Lee, H. C. \& Wei, Y. H. Oxidative stress, mitochondrial DNA mutation, and apoptosis in aging. Exp Biol Med (Maywood) 232, 592-606 (2007).

34. O’Donovan, D. J. \& Fernandes, C. J. Mitochondrial glutathione and oxidative stress: implications for pulmonary oxygen toxicity in premature infants. Mol Genet Metab 71, 352-358, https://doi.org/10.1006/mgme.2000.3063 (2000).

35. Buccellato, L. J., Tso, M., Akinci, O. I., Chandel, N. S. \& Budinger, G. R. Reactive oxygen species are required for hyperoxia-induced Bax activation and cell death in alveolar epithelial cells. The Journal of biological chemistry 279, 6753-6760, https://doi.org/10.1074/ jbc.M310145200 (2004).

36. Serviddio, G. et al. Uncoupling protein-2 (UCP2) induces mitochondrial proton leak and increases susceptibility of non-alcoholic steatohepatitis (NASH) liver to ischaemia-reperfusion injury. Gut 57, 957-965, https://doi.org/10.1136/gut.2007.147496 (2008).

37. Jezek, P., Holendova, B., Garlid, K. D. \& Jaburek, M. Mitochondrial Uncoupling Proteins: Subtle Regulators of Cellular Redox Signaling. Antioxid Redox Signal 29, 667-714, https://doi.org/10.1089/ars.2017.7225 (2018).

38. Brookes, P. S. Mitochondrial H(+) leak and ROS generation: an odd couple. Free Radic Biol Med 38, 12-23, https://doi.org/10.1016/j. freeradbiomed.2004.10.016 (2005).

39. Korzeniewski, B. Contribution of proton leak to oxygen consumption in skeletal muscle during intense exercise is very low despite large contribution at rest. PloS one 12, e0185991, https://doi.org/10.1371/journal.pone.0185991 (2017).

40. Grad, L. I. \& Lemire, B. D. Mitochondrial complex I mutations in Caenorhabditis elegans produce cytochrome coxidase deficiency, oxidative stress and vitamin-responsive lactic acidosis. Human molecular genetics 13, 303-314, https://doi.org/10.1093/hmg/ddh027 (2004).

41. Han, D., Williams, E. \& Cadenas, E. Mitochondrial respiratory chain-dependent generation of superoxide anion and its release into the intermembrane space. Biochem J 353, 411-416 (2001).

42. Lenaz, G. et al. Mitochondrial Complex I: structural and functional aspects. Biochim Biophys Acta 1757, 1406-1420, https://doi. org/10.1016/j.bbabio.2006.05.007 (2006)

43. Fato, R., Bergamini, C., Leoni, S., Strocchi, P. \& Lenaz, G. Generation of reactive oxygen species by mitochondrial complex I: implications in neurodegeneration. Neurochem Res 33, 2487-2501, https://doi.org/10.1007/s11064-008-9747-0 (2008).

44. Francis, B. M. et al. Reduced levels of mitochondrial complex I subunit NDUFB8 and linked complex I + III oxidoreductase activity in the TgCRND8 mouse model of Alzheimer's disease. Journal of Alzheimer's disease: JAD 39, 347-355, https://doi.org/10.3233/ JAD-131499 (2014)

45. Demarest, T. G. \& McCarthy, M. M. Sex differences in mitochondrial (dys)function: Implications for neuroprotection. J Bioenerg Biomembr 47, 173-188, https://doi.org/10.1007/s10863-014-9583-7 (2015).

46. Pinto, R. E. \& Bartley, W. The nature of the sex-linked differences in glutathione peroxidase activity and aerobic oxidation of glutathione in male and female rat liver. Biochem J 115, 449-456 (1969).

47. McCullough, L. D., Zeng, Z., Blizzard, K. K., Debchoudhury, I. \& Hurn, P. D. Ischemic nitric oxide and poly (ADP-ribose) polymerase-1 in cerebral ischemia: male toxicity, female protection. Journal of cerebral blood flow and metabolism: official journal of the International Society of Cerebral Blood Flow and Metabolism 25, 502-512, https://doi.org/10.1038/sj.jcbfm.9600059 (2005).

48. Bolisetty, S. et al. Neurodevelopmental outcomes of extremely preterm infants in New South Wales and the Australian Capital Territory. J Paediatr Child Health, https://doi.org/10.1111/jpc.14323 (2018).

49. Fombonne, E. Epidemiological surveys of autism and other pervasive developmental disorders: an update. J Autism Dev Disord 33, 365-382 (2003).

50. Vigelso, A., Andersen, N. B. \& Dela, F. The relationship between skeletal muscle mitochondrial citrate synthase activity and whole body oxygen uptake adaptations in response to exercise training. Int J Physiol Pathophysiol Pharmacol 6, 84-101 (2014)

51. Schieber, M. \& Chandel, N. S. ROS function in redox signaling and oxidative stress. Current biology: CB 24, R453-462, https://doi. org/10.1016/j.cub.2014.03.034 (2014).

52. Dargaei, Z. et al. Restoring GABAergic inhibition rescues memory deficits in a Huntington's disease mouse model. Proceedings of the National Academy of Sciences of the United States of America 115, E1618-E1626, https://doi.org/10.1073/pnas.1716871115 (2018).

53. Bilgel, M. et al. Effects of amyloid pathology and neurodegeneration on cognitive change in cognitively normal adults. Brain: $a$ journal of neurology 141, 2475-2485, https://doi.org/10.1093/brain/awy150 (2018).

54. Jilling, T. et al. Exposure of Neonatal Mice to Bromine Impairs Their Alveolar Development and Lung Function. American journal of physiology. Lung cellular and molecular physiology, ajplung 00315 02017, https://doi.org/10.1152/ajplung.00315.2017 (2017).

55. Mi, H. et al. PANTHER version 11: expanded annotation data from Gene Ontology and Reactome pathways, and data analysis tool enhancements. Nucleic Acids Res 45, D183-D189, https://doi.org/10.1093/nar/gkw1138 (2017).

56. Thomsen, K. et al. Initial brain aging: heterogeneity of mitochondrial size is associated with decline in complex I-linked respiration in cortex and hippocampus. Neurobiol Aging 61, 215-224, https://doi.org/10.1016/j.neurobiolaging.2017.08.004 (2018).

57. Janssen, A. J. et al. Spectrophotometric assay for complex I of the respiratory chain in tissue samples and cultured fibroblasts. Clin Chem 53, 729-734, https://doi.org/10.1373/clinchem.2006.078873 (2007).

58. Ramachandran, A. et al. Inhibition of mitochondrial protein synthesis results in increased endothelial cell susceptibility to nitric oxide-induced apoptosis. Proceedings of the National Academy of Sciences of the United States of America 99, 6643-6648, https://doi. org/10.1073/pnas.102019899 (2002).

59. Shepherd, D. \& Garland, P. B. The kinetic properties of citrate synthase from rat liver mitochondria. Biochem J 114, 597-610 (1969).

60. Fetterman, J. L. et al. Developmental exposure to second-hand smoke increases adult atherogenesis and alters mitochondrial DNA copy number and deletions in apoE(-/-) mice. PloS one 8, e66835, https://doi.org/10.1371/journal.pone.0066835 (2013).

\section{Acknowledgements}

The authors wish to thank the UAB BioAnalytical Redox Biology Core (P30DK079626) and Dr. Douglas Moellering for assistance in the mitochondrial functional measures. This work was partially funded by R01HL092906, R01AG021612, R01NS076312, R25NS089463, and the Kaul Pediatric Research Institute, Division of Neonatology, Department of Pediatrics, University of Alabama at Birmingham, Alabama USA.

\section{Author Contributions}

Concept and design: M.R., L.L.M., S.W.B. and N.A.; Data analysis and interpretation: M.R., R.K., J.K., L.L.M., S.W.B. and N.A.; Drafting the manuscript for important intellectual content: M.R., S.W.B. and N.A. Performed the research: M.R., K.M. and J.B. All authors edited and approved the final version of the manuscript.

Additional Information

Supplementary information accompanies this paper at https://doi.org/10.1038/s41598-019-49532-z. 
Competing Interests: The authors declare no competing interests.

Publisher's note: Springer Nature remains neutral with regard to jurisdictional claims in published maps and institutional affiliations.

(c) (i) Open Access This article is licensed under a Creative Commons Attribution 4.0 International License, which permits use, sharing, adaptation, distribution and reproduction in any medium or format, as long as you give appropriate credit to the original author(s) and the source, provide a link to the Creative Commons license, and indicate if changes were made. The images or other third party material in this article are included in the article's Creative Commons license, unless indicated otherwise in a credit line to the material. If material is not included in the article's Creative Commons license and your intended use is not permitted by statutory regulation or exceeds the permitted use, you will need to obtain permission directly from the copyright holder. To view a copy of this license, visit http://creativecommons.org/licenses/by/4.0/.

(C) The Author(s) 2019 